Original Research Paper

\title{
Introgression the SalTol QTL into the Elite Rice Variety of Russia by Marker-Assisted Selection
}

\author{
${ }^{1}$ Alexander Vyacheslavovich Usatov, ${ }^{2}$ Andrey Vasilevich Alabushev, ${ }^{2}$ Pavel Ivanovich Kostylev, \\ ${ }^{1}$ Kirill Vitalievich Azarin, ${ }^{1}$ Maxim Stanislavovich Makarenko and ${ }^{1}$ Olga Arturovna Usatova \\ ${ }^{I}$ Southern Federal University, Rostov-on-Don, Russia \\ ${ }^{2}$ All-Russian Research Institute of Grain Crops, I.G. Kalinenko, Zernograd, Russia
}

Article history

Received: 3-11-2015

Revised: 9-11- 2015

Accepted: 14-11-2015

Corresponding Author:

Alexander Vyacheslavovich

Usatov

Southern Federal University,

Rostov-on-Don, Russia

Email: azkir@rambler.ru

\begin{abstract}
The present work is devoted to investigation of the possibility to apply methods of Marker-Assisted Selection (MAS) to introgression the SalTol QTL into genotypes of elite Russian varieties of rice. It was shown that microsatellite markers Rm493, may be effectively used to control transfer the SalTol QTL genes into Russian populations of rice. Based on the highly productive variety "Novator", we obtained the lines Nov-129 and Nov-148 carrying loci SalTol in homozygous state. The lines Nov-129 and Nov-148 are used as an improved salt tolerance donor source to obtain hybrids tolerance to salinity.
\end{abstract}

Keywords: SalTol, DNA-Markers, Marker-Assisted Selection, Rice

\section{Introduction}

At the present time, new technologies that allow to reduce the period of selection process and to increase reliability of breeding material analysis is particularly important for breeding crops. One of these approaches is the technology of molecular markers (Hospital, 2009; Kumar et al., 2015). DNA marker analyses provide several significant advantages in comparison with analysis of morphological and physiological traits (Collard et al., 2008; Usatov et al., 2014a). Almost any gene or locus may be marked, suggesting the existence of a number of such specific markers (Hospital, 2009; Platten et al., 2013). In particular, DNA markers may be successfully used in breeding of rice with respect to its tolerance to salinity (Ashraf et al., 2012).

Soil salinity is one of the major problems in all over the World. Presently, a major QTL SalTol associated with the Na-K ratio in rice and seedlingstage salinity tolerance, are marked on chromosome 1 (Platten et al., 2013). Nevertheless, allelic variations of microsatellite loci associated with specific trait in related groups of genotypes do not always correlate with that trait in genotypes other origins (Senadheera et al., 2009; Mardani et al., 2014). This circumstance can lead to erroneous results when analyzing the presence of the recipient alleles in hybrid population (Ashraf et al., 2012; Mardani et al., 2014). In this connection, the study of the informational value of SSR markers associated with SalTol QTL for introgression the
SalTol region into genotype of the elite Russian variety of rice was conducted.

\section{Materials and Methods}

\section{Plant Materials}

The highly productive elite variety "Novator", which was obtained from the I. G. Kalinenko All-Russian Institute for Crop Cultures (VNIIZK) (Russia) was used as the recipient. Line IR61920-3B-22-2-1 (NSIC Rc106) was used as a donor SalTol region.

\section{Genotyping}

SalTol region was identified during the selection process by closely linked microsatellite markers RM8094, RM493 (http://gramene.com).

To perform the molecular genetic analysis, genomic DNA was isolated from leaf tissue as described in (Boom et al., 1990), with our modification (Markin et al., 2015). Polymerase chain reaction was carried out in $25 \mu \mathrm{L}$ reaction mixture of the following composition: $67 \mathrm{~mm}$ Tris- $\mathrm{HCl}$ buffer, $\mathrm{pH}$ 8.8, $16 \mathrm{mM}\left(\mathrm{NH}_{4}\right)_{2} \mathrm{SO}_{4}, 2.5 \mathrm{mM} \mathrm{MgSO}, 0.1 \mathrm{mM}$ mercaptoethanol, $0.25 \mathrm{mM}$ of each dNTP (dATP, dCTP, dTTP and dGTP), 20 pM primers, 2.5 units of Taqpolymerase and $15 \mathrm{ng}$ isolated DNA. Amplification was performed in the thermo cycler Palm Cycler (Corbett Research, Australia). Thermal regime of the reaction was 
chosen individually for each pair of primers on the basis of their sequences. For majority of reactions the optimal thermal regime was as follows: (1) denaturation at $94^{\circ} \mathrm{C}$ for $5 \mathrm{~min}$, (2) 35 cycles at the following thermal and time regime: primer annealing at $55-60^{\circ} \mathrm{C}$ for $30 \mathrm{~s}, 30 \mathrm{~s}$ elongation at $72^{\circ} \mathrm{C}$, denaturation at $94^{\circ} \mathrm{C}, 30 \mathrm{~s}$, (3) $8 \mathrm{~min}$ final elongation at $72^{\circ} \mathrm{C}$ (Usatov et al., 2014b). Amplification products were analyzed by electrophoresis in $2 \%$ agarose gel supplemented with ethidium bromide in Tris-Borate buffer. The obtained gels were photographed with the Gel-Documenting system (GelDoc 2000, BioRad, United States). Gene Ruler $1 \mathrm{~Kb}$ DNA Ladder (Fermentas, Lithuania) was used as a molecular weight marker.

\section{Evaluation of Salinity Tolerance}

In a laboratory experiment, rice seeds were soaked in water for $12 \mathrm{hrs}$, then placed in special trays and saline added in concentrations of $1 \% \mathrm{NaCl}$ (Thomson et al., 2010; Xiong and Choong, 2014). Control seeds were grown in distilled water. Seedlings were grown in a growth chamber (KBWF E5.2; Binder, GmbH) under the following controlled environmental conditions: $70 \%$ relative humidity, $26 \pm 2 \mathrm{C}$ and a $14 \mathrm{~h}$ photoperiod. After 14 days, root length, shoot length and total germination percentage were measured (Ali et al., 2014; Kumar and Kumar, 2014).

\section{Determining Concentrations of Sodium and Potassium}

The $\mathrm{Na}+$ and $\mathrm{K}+$ content in shoots and roots were measured using a capillary electrophoresis system (Capel-105M; Lumex, Russia) according to the method of Platten et al. (2013).

Dried samples of plant material were treated with 10 $\mathrm{mL}$ deionized water at $100^{\circ} \mathrm{C}$ for $1 \mathrm{~h}$ and the extract used to determine the contents of free inorganic ions.

\section{Statistical Analysis}

The collected data were subjected to Analysis Of Variance (ANOVA). All data were represented by an average of the ten biological replicates and the Standard Deviations (SD).

\section{Results}

\section{Parental SSR Polymorphism Screening}

In this study, 21 SSR markers associated with the SalTol/QTL region were checked with two parent's varieties in order to find out polymorphic primers to further use for screening the Saltol loci of the crossing populations. The molecular analysis of rice samples by 2 microsatellite markers of the SalTol loci showed that only $\mathrm{Rm} 493$ provided reliable well-reproducible spectra and in formativeness for identification of the SalTol region in hybrid population.

\section{Genotyping}

We performed hybridization of the conventional variety Novator, subspecies japonica and sensitive to salt with the line NSIC Rc106, subspecies indica, which was used as a donor of the SalTol loci, in order to develop tolerant lines of rice on the basis of Russian varieties. First generation hybrids were used for obtainment of $F_{2}$ population.

Out of the $F_{2}$ generation plants by the traits of earliness, non-shattering of seeds and exaristate were selected 90 rice plants. Selected plants were analyzed by PCR for the presence of introduced alleles SalTol. Data of electrophoretic analysis of PCR product of the Rm493 marker, which is linked to the SalTol, shown on Fig. 1.

Donor tolerance allele of the parental line NSIC Rc106, which is marked on the Fig. 1 as 2.2, was found in homozygous state in two samples (129 and 148). Plants No. 62-64, 66, 67, 138-147, 149 and 150 carried both donor and maternal alleles, which were heterozygous by the SalTol locus. Other samples carried only the allele inherited from the variety "Novator" and thus, were rejected.

\section{Evaluation of Salinity Tolerance}

Evaluation of potential salt tolerance of the $F_{2}$ rice plants at seedling stage revealed significant variations of tolerance to salinity depending on the genotype (Table 1).

Sensitive to salt variety Novator was characterized by the highest decline seed germination $-55,6 \%$. The line NSIC Rc106 and second generation plants which were homo-, heterozygous by locus SalTol showed the most stable by traits of germination (decrease germination less than 5\%).

The lowest suppression of growth parameters was showen in the line NSIC Rc106 and homozygous by SalTol fragment of $\mathrm{F}_{2}$ generation plants, while the largest decline roots length and shoots height under salt stress was found in the variety "Novator" and second generation plants that not inherited SalTol locus according to the data of molecular analysis (Table 1).

\section{Potassium/Sodium Content Ratio}

The $\mathrm{K}+/ \mathrm{Na}+$ content ratio in shoot and root of the studied rice varieties is shown in the Table 2 .

In general, it was found that by applying salinity, the $\mathrm{K}+/ \mathrm{Na}+$ ratio in root was significantly $(\mathrm{p} \leq 0.01)$ reduced both in parental varieties and $F_{2}$ generation plants. On the other hand, the saline-susceptible variety Novator and $\mathrm{F}_{2}$ generation plants without of the SalTol fragment had a $\mathrm{K}+/ \mathrm{Na}+$ ratio in shoot significantly less than the line NSIC Rc106 and $F_{2}$ generation plants carrying the SalTol loci (tolerant). 


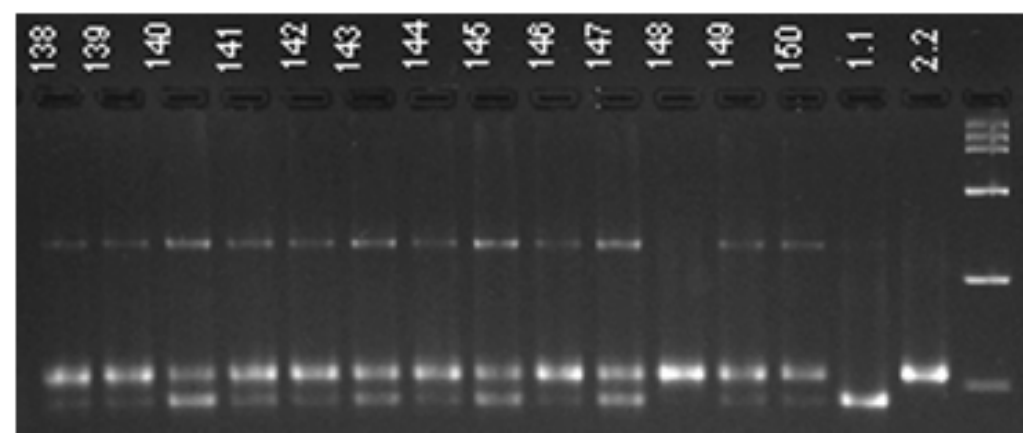

Fig. 1. The electrophoregram of amplification products of genomic DNA of rice with the primer Rm493. Molecular weight marker $1 \mathrm{~Kb}$

Table 1. The germination (\%), shoot length $(\mathrm{cm})$ and root length $(\mathrm{cm})$ of 14-day-old seedlings of rice under control and salt stress conditions

\begin{tabular}{|c|c|c|c|c|c|c|}
\hline \multirow[b]{2}{*}{ Samples } & \multicolumn{2}{|c|}{ Germination (\%) } & \multicolumn{2}{|c|}{ Shoot length $(\mathrm{cm})$} & \multicolumn{2}{|c|}{ Root length (cm) } \\
\hline & Control & $1 \% \mathrm{NaCl}$ & Control & $1 \% \mathrm{NaCl}$ & Control & $1 \% \mathrm{NaCl}$ \\
\hline Novator & $99 \pm 1$ & $52 \pm 2.5^{*}$ & $11.4 \pm 1.3$ & $3.4 \pm 0.5^{*}$ & $9.3 \pm 1.0$ & $2.2 \pm 0.7^{*}$ \\
\hline NSIC Rc106 & $87 \pm 1.5$ & $85 \pm 1$ & $13.6 \pm 1.6$ & $7.2 \pm 1.1$ & $11.5 \pm 1.7$ & $7.7 \pm 1.0$ \\
\hline 129 & $85 \pm 2$ & $85 \pm 1$ & $14.3 \pm 1.5$ & $7.5 \pm 0.9$ & $10.1 \pm 0.8$ & $6.7 \pm 0.9$ \\
\hline 148 & $90 \pm 3$ & $85 \pm 2$ & $14.9 \pm 1.5$ & $8.1 \pm 1.2$ & $10.5 \pm 0.5$ & $6.3 \pm .1$ \\
\hline Hetero-zygotes & $85 \pm 1$ & $87 \pm 1.5$ & $13.2 \pm 2.3$ & $6.2 \pm 1.8$ & $10.8 \pm 1.5$ & $5.1 \pm 0.9$ \\
\hline $\begin{array}{l}\text { Homo-zygotes } \\
\text { (withoutSalTol) }\end{array}$ & $94 \pm 1.5$ & $61 \pm 2 *$ & $12.2 \pm 1.2$ & $4.6 \pm 0.6^{*}$ & $9.7 \pm 1.4$ & $1.69 \pm 0.9 *$ \\
\hline
\end{tabular}

*Indicate significant differences from control at $\mathrm{p}<0.001$

Table 2. $\mathrm{K}+/ \mathrm{Na}+$ ratio in shoots and roots of 14-day-old seedlings of rice under control and salt stress conditions

\begin{tabular}{|c|c|c|c|c|}
\hline \multirow[b]{2}{*}{ Samples } & \multicolumn{2}{|l|}{ Shoot } & \multicolumn{2}{|l|}{ Root } \\
\hline & Control & $1 \% \mathrm{NaCl}$ & Control & $1 \% \mathrm{NaCl}$ \\
\hline Novator & $0.9 \pm 0.13$ & $0.20 \pm 0.05^{*}$ & $0.46 \pm 0.05$ & $0.21 \pm 0.07^{*}$ \\
\hline NSIC Rc106 & $1.77 \pm 0.17$ & $1.62 \pm 0.13$ & $1.60 \pm 0.23$ & $0.19 \pm 0.02 *$ \\
\hline 129 & $1.73 \pm 0.15$ & $1.63 \pm 0.21$ & $1.82 \pm 0.20$ & $0.66 \pm 0.08$ \\
\hline 148 & $2.01 \pm 0.19$ & $1.80 \pm 0.10$ & $1.5 \pm 0.17$ & $0.75 \pm 0.04^{*}$ \\
\hline Hetero-zygotes & $1.57 \pm 0.13$ & $1.45 \pm 0.16$ & $1.71 \pm 0.15$ & $0.69 \pm 0.09 *$ \\
\hline $\begin{array}{l}\text { Homo-zygotes } \\
\text { (withoutSalTol) }\end{array}$ & $1.18 \pm 0.03$ & $0.34 \pm 0.15^{*}$ & $0.39 \pm 0.03$ & $0.18 \pm 0.11^{*}$ \\
\hline
\end{tabular}

\section{Discussion}

The $F_{1}$ progeny obtained from the crossbreeding "Novator" $\times$ NSIC Rc106 may be use in a series of backcrosses, which provided introduction of donor resistance alleles into the genotype of the recurrent parental form (the variety Novator) (Wang et al., 2011). Presence of the transferred alleles should be assessed by the method of MAS as described above. In the present work we selected perspective lines, which resembled the parental recipient phenotype, from the $\mathrm{F}_{2}$ population obtained by self-pollination.

PCR analysis of linked markers allowed us to identify $\mathrm{F}_{2}$ plants that carried different allelic variants of the SalTol loci. Samples 129 (Novator $\times$ NSIC Rc106) and 148 (Novator $\times$ NSIC Rc106) were homozygous by SalTol loci. Moreover, we identified fifteen lines that carried SalTol loci in heterozygous.

Laboratory testing of the breeding material of rice showed that germination, root length and shoot length at seedling stage decreased under salt stress. The lowest inhibition of growth processes under salinity was observed in the line NSIC Rc106 which was used as donor of the SalTol loci and in the $\mathrm{F}_{2}$ plants having been inherited SalTol locus according to the SSR analysis. Determining concentrations of sodium and potassium in root and shoot revealed shoot $\mathrm{K}+/ \mathrm{Na}+$ homeostasis in these lines, that is the evidence of working Saltol fragment. The data presented here is similar to the results of other studies (Mardani et al., 2014; Negrão et al., 2011).

Newly developed lines 129 (Novator $\times$ NSIC Rc106) and 148 (Novator $\times$ NSIC Rc106), carrying SalTol loci in 
homozygous state, are used as the improved donor source of salt tolerance in order to obtain heterosis hybrids tolerant to the salinity.

It was previously shown that the salt tolerance of rice is the genetics of quantitative traits, which is controlled by multiple genes (Negrão et al., 2011; Mekawy et al., 2011). It had mapped more than 70 QTLs which were linked to the salt tolerance and two salt tolerance genes of rice (SKC1 and DST) have already been cloned ( $\mathrm{Hu}$ et al., 2012; Cotsaftis et al., 2012; Kumar et al., 2015). Introduction of the tolerance QTLs into the elite varieties adapted to certain agro-climatic conditions, as well as pyramiding of several tolerance QTLs in one genotype are considered to be the most perspective ways of selection of varieties tolerant to the salt stress (Ashraf et al., 2012; Das et al., 2015; Hoang et al., 2015).

Marker Assisted Selection (MAS) as a high technology tool is presently increasingly used for real selection programs (Collard et al., 2008; Markin et al., 2015). Application of MAS provides clear understanding of the targeted gene inheritance from parental lines (Kumar et al., 2015). Therefore, it may be effectively used to correct the breeding of plants in order to obtain of new varieties less cost and time consuming (Wang et al., 2011; Kumar et al., 2015).

Nevertheless, only integration of molecular genotyping data with classical complex analysis of morphophysiological traits of plants both in laboratory model experiments and in field are required to solve the problems of genetic marking of agronomic characters of crops.

\section{Conclusion}

The present study aimed at the application of marker assisted selection to introgression of the salinity tolerance QTLs SalTol into the genotypes of elite Russian varieties of rice. It was shown that Rm493 microsatellite markers may be effectively used to control the transfer of the SalTol fragment into the Russian populations of rice. Individual selection by morphological traits and in combination with MAS allowed us to simplify the selection scheme and obtain samples, carrying SalTol locus in homozygous condition. These samples were characterized by a complex of traits that corresponded to the agro-climatic conditions for rice breeding in Russia. This selection material was used as the improved source of tolerance to salinity in order to obtain heterosis hybrids tolerance to salt stress.

\section{Acknowledgement}

This research was supported by the Russian Ministry of Education and Science, project no. 40.91.2014/K.

\section{Funding Information}

The funders of this manuscript were the Ministry of Education and Science of the Russian Federation.

\section{Author's Contributions}

All the six authors participated in the laboratory study, data analysis and the entire process of the article preparation.

\section{Ethics}

The authors state that this article conforms to the ethical standards specified by the American Journal of Agricultural and Biological Sciences.

\section{References}

Ali, Md. N., L. Yeasmin, S. Gantait, R. Goswami and S. Chakraborty, 2014. Screening of rice landraces for salinity tolerance at seedling stage through morphological and molecular markers. Physiol. Mol. Biol. Plants, 20: 411-423.

DOI: $10.1007 / \mathrm{s} 12298-014-0250-6$

Ashraf, M., N.A. Akram, Mehboob-Ur-Rahman and M.R. Foolad, 2012. Marker-assisted selection in plant breeding for salinity tolerance. Methods Mol. Bio., 913: 305-333. DOI: 10.1007/978-1-61779-986-0 21

Boom, R., C.J. Sol, M.M. Salimans, C.L. Jansen and P.M. Wertheim-van Dillen et al., 1990. Rapid and simple method for purification of nucleic acids. J. Clin. Microbiol., 28: 495-503. DOI: 0095-1137/90/030495-09\$02.00/0

Collard, B.C. and D.J. Mackill, 2008. Marker-assisted selection: An approach for precision plant breeding in the twenty-first century. Philo. Trans. B, 363: 557-572. DOI: 10.1098/rstb.2007.2170

Cotsaftis, O., D. Plett, N. Shirley, M. Tester and M. Hrmova, 2012. A two-staged model of $\mathrm{Na}+$ exclusion in rice explained by $3 \mathrm{D}$ modeling of $\mathrm{HKT}$ transporters and alternative splicing. PLoS ONE, DOI: 10.1371/journal.pone.0039865

Das, P., K.K. Nutan, S.L. Singla-Pareek and A. Pareek, 2015. Understanding salinity responses and adopting "omics-based" approaches to generate salinity tolerant cultivars of rice. Frontiers Plant Sci. DOI: $10.3389 /$ fpls.2015.00712

Hoang, T.M.L., L. Moghaddam, B. Williams, H. Khanna and J. Dale et al., 2015. Development of salinity tolerance in rice by constitutive-over expression of genes involved in the regulation of programmed cell death. Frontiers Plant Sci. DOI: $10.3389 /$ fpls.2015.00175

Hospital, F., 2009. Challenges for effective markerassisted selection in plants. Genetica, 136: 303-310. DOI: $10.1007 / \mathrm{s} 10709-008-9307-1$ 
Hu, S., H. Tao, Q. Qian and L. Guo, 2012. Genetics and molecular breeding for salt-tolerance in rice. Rice Genomics Genetics, 3: 38-39. DOI: $10.5376 /$ rgg.2012.03.0007

Kumar, D. and R.V. Kumar, 2014. Efficacy of bio-foliar spray on growth and biochemical parameters of different mulberry varieties. OnLine J. Biol. Sci., 14: 64-69. DOI: 10.3844/ojbsci.2014.64.69

Kumar, V., A. Singh, S.V.A. Mithra, S.L. Krishnamurthy and S.K. Parida et al., 2015. Genome-wide association mapping of salinity tolerance in rice (Oryza sativa). DNA Research: An Int. J. Rapid Pub. Reports Genes Genomes, 22: 133-145. DOI: 10.1093/dnares/dsu046

Mardani, Z., B. Rabiei, H. Sabouri and A. Sabouri, 2014. Identification of molecular markers linked to salttolerant genes at germination stage of rice. Plant Breeding, 133: 196-202. DOI: 10.1111/pbr.12136

Markin, N.V., A.V. Usatov, M.D. Logacheva, K.V. Azarin and O.F. Gorbachenko et al., 2015. Study of chloroplast DNA polymorphism in the sunflower (Helianthus L.). Russian J. Genetics, 51: 745-751. DOI: $10.1134 / \mathrm{S} 1022795415060101$

Mekawy, A.M., D.V. Assaha, H. Yahagi, Y. Tada and A. Ueda et al., 2011. Growth, physiological adaptation and gene expression analysis of two Egyptian rice cultivars under salt stress. Plant Physiology Biochem., 87:

$17-25$. DOI: 10.1016/j.plaphy.2014.12.007

Negrão, S., B. Courtois, N. Ahmadi, I. Abreu and N. Saibo et al., 2011. Recent updates on salinity stress in rice: From physiological to molecular responses. Critical Rev. Plant Sci., 30: 329-377. DOI: $10.1080 / 07352689.2011 .587725$
Platten, J.D., J.A. Egdane and A.M. Ismail, 2013. Salinity tolerance, $\mathrm{Na}+$ exclusion and allele mining of HKT1; 5 in Oryza sativa and O. glaberrima: Many sources, many genes, one mechanism. BMC Plant Biol. DOI: 10.1186/1471-2229-13-32

Senadheera, P., R.K. Singh and F.J.M. Maathuis, 2009. Differentially expressed membrane transporters in rice roots may contribute to cultivar dependent salt tolerance. J. Exper. Botany, 60: 2553-2563. DOI: $10.1093 / \mathrm{jxb} / \mathrm{erp} 099$

Thomson, M.J., M. Ocampo, J. Egdane, M.A. Rahman and A.G. Sajise et al., 2010. Characterizing the Saltol quantitative trait locus for salinity tolerance in rice. Rice, 3: 148-160. DOI: 10.1007/s12284-010-9053-8

Usatov, A.V., A.I. Klimenko, K.V. Azarin, O.F. Gorbachenko and N.V. Markin, 2014a. The relationship between heterosis and genetic distances based on SSR markers in Helianthus annuus. Am. J. Agric. Biol. Sci., 9: 270-276. DOI: 10.3844/ajabssp.2014.270.276

Usatov, A.V., A.I. Klimenko, K.V. Azarin, O.F. Gorbachenko and N.V. Markin et al., 2014b. DNAmarkers of sunflower resistance to the downy mildew (Plasmopara halstedii). Am. J. Biochem. Biotech., 10: 125-129. DOI: $10.3844 /$ ajbbsp.2014.125.129

Wang, Z., J. Wang, Y. Bao, F. Wang and Y. Wu et al., 2011. Quantitative trait loci controlling rice seed germination under salt stress. Euphytica, 178: 297-307. DOI: $10.1007 /$ s10681-010-0287-8

Xiong, Z. and C.W. Choong, 2014. Optimum micronutrient level for Phalaenopsis deliciosa orchid seedling In vitro growth. Online J. Biol. Sci., 14: 240-247. DOI: 10.3844/ojbsci.2014.240.247 